\title{
Solvability of a Fourth-Order Boundary Value Problem with Integral Boundary Conditions
}

\author{
Hui Li, Libo Wang, and Minghe Pei \\ Department of Mathematics, Beihua University, Jilin City 132013, China \\ Correspondence should be addressed to Minghe Pei; peiminghe@163.com \\ Received 21 December 2012; Accepted 13 February 2013 \\ Academic Editor: Meng Fan
}

Copyright (C) 2013 Hui Li et al. This is an open access article distributed under the Creative Commons Attribution License, which permits unrestricted use, distribution, and reproduction in any medium, provided the original work is properly cited.

We investigate the existence of solutions and positive solutions for a nonlinear fourth-order differential equation with integral boundary conditions of the form $x^{(4)}(t)=f\left(t, x(t), x^{\prime}(t), x^{\prime \prime}(t), x^{\prime \prime \prime}(t)\right), t \in[0,1], x(0)=x^{\prime}(1)=0, x^{\prime \prime}(0)=\int_{0}^{1} h(s, x(s)$, $\left.x^{\prime}(s), x^{\prime \prime}(s)\right) \mathrm{d} s, x^{\prime \prime \prime}(1)=0$, where $f \in C\left([0,1] \times \mathbb{R}^{4}\right), h \in C\left([0,1] \times \mathbb{R}^{3}\right)$. By using a fixed point theorem due to D. O'Regan, the existence of solutions and positive solutions for the previous boundary value problems is obtained. Meanwhile, as applications, some examples are given to illustrate our results.

\section{Introduction}

It is well known that fourth-order boundary value problems (BVPs) arise in a variety of different areas of the flexibility mechanics and engineering physics and thus have been extensively studied; for instance, see [1-29] and references therein. Boundary value problems with integral boundary conditions appear in heat conduction, thermoelasticity, chemical engineering underground water flow, and plasma physics; see [12, $14,21,24,26,29]$ and references therein.

Motivated by the previous works and [30], in this paper, we consider fully nonlinear fourth-order differential equation

$$
x^{(4)}(t)=f\left(t, x(t), x^{\prime}(t), x^{\prime \prime}(t), x^{\prime \prime \prime}(t)\right), \quad t \in[0,1],
$$

subject to the integral boundary conditions

$$
\begin{gathered}
x(0)=x^{\prime}(1)=0, \\
x^{\prime \prime}(0)=\int_{0}^{1} h\left(s, x(s), x^{\prime}(s), x^{\prime \prime}(s)\right) \mathrm{d} s, \\
x^{\prime \prime \prime}(1)=0,
\end{gathered}
$$

as well as its simplified form

$$
x^{(4)}(t)=f(t, x(t)), \quad t \in[0,1],
$$

with the integral boundary conditions

$$
\begin{gathered}
x(0)=x^{\prime}(1)=0, \\
x^{\prime \prime}(0)=\int_{0}^{1} h(s, x(s)) \mathrm{d} s, \\
x^{\prime \prime \prime}(1)=0,
\end{gathered}
$$

where $f$ and $h$ are continuous functions.

We notice that if $h \equiv 0$ in problems (1), (2) and (3), (4), then the models are known as the one endpoint simply supported and the other one sliding clamped beam. The study of this class of problems was considered by some authors via various methods; we refer the reader to the papers $[2,5,8$, $11,22]$.

The aim of this paper is to establish the existence results of solutions and positive solutions for problems (1), (2) and (3), (4), respectively. By positive solution, we mean a solution $x(t)$ such that $x(t)>0$ for $t \in(0,1]$. Our main tool is the fixed point theorem due to D. O'Regan [31].

\section{Preliminary}

In this section, we present some lemmas which are needed for our main results. 
Let $C[0,1]$ denote the Banach space of real-valued continuous functions on $[0,1]$ with the norm $\|x\|_{0}:=\max _{t \in[0,1]}|x(t)|$. $C^{n}[0,1]$ is the Banach space of $n$ times continuously differentiable functions defined on $[0,1]$, with the norm $\|x\|_{C^{n}}:=$ $\max \left\{\left\|x^{(i)}\right\|_{0}, i=0,1, \ldots, n\right\}$.

Throughout this paper, we always assume that $f:[0,1] \times$ $\mathbb{R}^{4} \rightarrow \mathbb{R}=(-\infty,+\infty)($ or $[0,1] \times \mathbb{R} \rightarrow \mathbb{R})$ and $h:[0,1] \times \mathbb{R}^{3} \rightarrow \mathbb{R}$ $($ or $[0,1] \times \mathbb{R} \rightarrow \mathbb{R}$ ) are continuous.

We consider a priori bound of solutions of the following one-parameter family of boundary value problem:

$$
\begin{gathered}
x^{(4)}(t)=\lambda f\left(t, x(t), x^{\prime}(t), x^{\prime \prime}(t), x^{\prime \prime \prime}(t)\right), \quad t \in[0,1], \\
x(0)=x^{\prime}(1)=0, \\
x^{\prime \prime}(0)=\lambda \int_{0}^{1} h\left(s, x(s), x^{\prime}(s), x^{\prime \prime}(s)\right) \mathrm{d} s, \\
x^{\prime \prime \prime}(1)=0,
\end{gathered}
$$

where $0 \leq \lambda \leq 1$. Simple computations lead to the following lemma.

Lemma 1. $B V P(5),(6)$ with $\lambda=0$ has only the trivial solution, and the corresponding Green function $G(t, s)$ exists and is given by

$$
G(t, s)= \begin{cases}\left(s-\frac{1}{2} s^{2}\right) t-\frac{t^{3}}{6}, & 0 \leq t \leq s \leq 1 \\ \left(s-\frac{1}{2} s^{2}\right) t-\frac{t^{3}}{6}+\frac{1}{6}(t-s)^{3}, & 0 \leq s \leq t \leq 1 .\end{cases}
$$
by

Now, define a linear operator $L: C^{4}[0,1] \rightarrow C[0,1] \times \mathbb{R}^{4}$

$$
\begin{array}{r}
(L x)(t)=\left(x^{(4)}(t), x(0), x^{\prime}(1), x^{\prime \prime}(0), x^{\prime \prime \prime}(1)\right), \\
t \in[0,1] .
\end{array}
$$

Then, we can easily show that $L$ is a Fredholm operator with index zero, and its inverse $L^{-1}: C[0,1] \times \mathbb{R}^{4} \rightarrow C^{4}[0,1]$ is given by

$$
\begin{aligned}
L^{-1}(y, a, b, c, d)(t)= & \int_{0}^{1} G(t, s) y(s) \mathrm{d} s+a+b t+c g(t) \\
& +\mathrm{d}\left(\frac{1}{6} t^{3}-\frac{1}{2} t\right), \quad t \in[0,1],
\end{aligned}
$$

where $g(t)=(1 / 2) t^{2}-t$.

Define Nemytskii operators $F: C^{3}[0,1] \rightarrow C[0,1]$ induced by $f$ as

$$
(F x)(t)=f\left(t, x(t), x^{\prime}(t), x^{\prime \prime}(t), x^{\prime \prime \prime}(t)\right), \quad t \in[0,1],
$$

and $H: C^{3}[0,1] \rightarrow C[0,1]$ induced by $h$ as

$$
(H x)(t)=h\left(t, x(t), x^{\prime}(t), x^{\prime \prime}(t)\right), \quad t \in[0,1] .
$$

Also, define an operator $T: C^{3}[0,1] \rightarrow C[0,1] \times \mathbb{R}^{4}$ as

$$
(T x)(t)=\left((F x)(t), 0,0, \int_{0}^{1}(H x)(s) \mathrm{d} s, 0\right) .
$$

Simple computations yield the following lemma.

Lemma 2. BVP (5), (6) is equivalent to the abstract equation

$$
x=\lambda L^{-1} T x
$$

in $C^{3}[0,1]$; that is, $x \in C^{4}[0,1]$ is a solution of $B V P(5)$, (6) if and only if $x \in C^{3}[0,1]$ is a solution of the integral equation

$$
x(t)=\lambda\left[\int_{0}^{1} G(t, s)(F x)(s) \mathrm{d} s+\int_{0}^{1} \varphi(t, x(s)) \mathrm{d} s\right],
$$

where $\varphi(t, x(s))=g(t)(H x)(s)$.

Let us denote the operators $P_{1}, P_{2}$ as

$$
\begin{gathered}
\left(P_{1} x\right)(t)=\int_{0}^{1} G(t, s)(F x)(s) \mathrm{d} s, \\
\left(P_{2} x\right)(t)=\int_{0}^{1} \varphi(t, x(s)) \mathrm{d} s .
\end{gathered}
$$

Then, $L^{-1} T$ can be written as

$$
L^{-1} T=P_{1}+P_{2} .
$$

Now, we can easily give some properties of the Green function $G(t, s)$ and $g(t)$ by direct computation.

Lemma 3. Let $G(t, s)$ be as in Lemma 1 and $g(t)=(1 / 2) t^{2}-t$. Then,

(1) $0 \leq G(t, s) \leq \max _{0 \leq t, s \leq 1} G(t, s)=1 / 3, t, s \in[0,1]$;

$$
\begin{aligned}
0 & \leq\left(\frac{\partial}{\partial t}\right) G(t, s) \\
& = \begin{cases}s-\left(\frac{1}{2}\right) s^{2}-\left(\frac{1}{2}\right) t^{2}, & 0 \leq t \leq s \leq 1 ; \\
s-t s, & 0 \leq s \leq t \leq 1 ;\end{cases} \\
0 & \geq\left(\frac{\partial^{2}}{\partial t^{2}}\right) G(t, s) \\
& = \begin{cases}-t, & 0 \leq t \leq s \leq 1 ; \\
-s, & 0 \leq s \leq t \leq 1 ;\end{cases}
\end{aligned}
$$

(2) $g(t) \leq 0, g^{\prime}(t) \leq 0, g^{\prime \prime}(t)=1, g^{\prime \prime \prime}(t)=0, t \in[0,1]$;

(3) $\|g\|_{0}=1 / 2,\left\|g^{\prime}\right\|_{0}=1,\left\|g^{\prime \prime}\right\|_{0}=1,\left\|g^{\prime \prime \prime}\right\|_{0}=0$, $\|g\|_{C^{3}}=1$. 


\section{Lemma 4. Suppose that}

(i) for each fixed $\left(t, x_{2}, x_{3}\right) \in[0,1] \times \mathbb{R}^{2}, f\left(t, x_{0}, x_{1}, x_{2}\right.$, $x_{3}$ ) is nondecreasing in $x_{0}$ and $x_{1}$;

(ii) there exists a constant $M>0$ such that for $|x|>M$, $t \in[0,1]$,

$$
x f(t,-x,-x, x, 0)>0
$$

(iii) there exist $\beta \in(0,1)$ and nondecreasing continuous function $\sigma:[0,+\infty) \rightarrow[0,+\infty)$ such that $\sigma(u) \leq \beta u$ for $u>0$, and

$$
\begin{aligned}
& \left|h\left(t, x_{0}, x_{1}, x_{2}\right)-h\left(t, y_{0}, y_{1}, y_{2}\right)\right| \\
& \quad \leq \sigma\left(\max \left\{\left|x_{i}-y_{i}\right|, i=0,1,2\right\}\right)
\end{aligned}
$$

for all $\left(t, x_{0}, x_{1}, x_{2}\right),\left(t, y_{0}, y_{1}, y_{2}\right) \in[0,1] \times \mathbb{R}^{3}$.

Then, any solution $x=x(t)$ of $B V P(5),(6)$ satisfies

$$
\left|x^{(i)}(t)\right| \leq M+r, \quad i=0,1,2, t \in[0,1],
$$

where $r=(1-\beta)^{-1}\|h(\cdot, 0,0,0)\|_{0}$.

Proof. Let us first show that

$$
\left|x^{\prime \prime}(t)\right| \leq M+r, \quad \forall t \in[0,1] .
$$

Note that if $\lambda=0$ in (5) and (6), then BVP (5), (6) has only the trivial solution, and thus (23) holds. Hence, we may assume that $\lambda \in[0,1]$. Suppose now that (23) is not true. Then, there exists $t_{0} \in[0,1]$ such that $\left|x^{\prime \prime}\left(t_{0}\right)\right|>M+r$. Let

$$
K:=\left|x^{\prime \prime}\left(t_{1}\right)\right|=\max _{t \in[0,1]}\left|x^{\prime \prime}(t)\right| .
$$

Then, $K>M+r$, and from $x(0)=x^{\prime}(1)=0$, we have

$$
\left|x^{(i)}(t)\right| \leq K, \quad i=0,1, \forall t \in[0,1] .
$$

It is easy to see that $t_{1} \in[0,1]$. In fact, if $t_{1}=0$, then $\left|x^{\prime \prime}(0)\right|=$ $K$. From (6) and (iii), it follows that for some $\zeta \in[0,1]$,

$$
\begin{aligned}
K=\left|x^{\prime \prime}(0)\right|= & \lambda\left|\int_{0}^{1} h\left(s, x(s), x^{\prime}(s), x^{\prime \prime}(s)\right) \mathrm{d} s\right| \\
\leq & \left|h\left(\zeta, x(\zeta), x^{\prime}(\zeta), x^{\prime \prime}(\zeta)\right)\right| \\
\leq & \left|h\left(\zeta, x(\zeta), x^{\prime}(\zeta), x^{\prime \prime}(\zeta)\right)-h(\zeta, 0,0,0)\right| \\
& +|h(\zeta, 0,0,0)| \\
\leq & \beta K+(1-\beta) r \\
< & \beta K+(1-\beta) K=K,
\end{aligned}
$$

which is a contradiction, and thus $t_{1} \in(0,1]$. Furthermore, by definition of $t_{1}$ and (6), we have $x^{\prime \prime \prime}\left(t_{1}\right)=0$. Hence, from assumptions (i) and (ii) and (25), we have

$$
\begin{gathered}
x^{\prime \prime}\left(t_{1}\right) x^{(4)}\left(t_{1}\right)=\lambda x^{\prime \prime}\left(t_{1}\right) f\left(t_{1}, x\left(t_{1}\right), x^{\prime}\left(t_{1}\right), x^{\prime \prime}\left(t_{1}\right), 0\right) \\
\geq \lambda x^{\prime \prime}\left(t_{1}\right) f\left(t_{1},-x^{\prime \prime}\left(t_{1}\right),-x^{\prime \prime}\left(t_{1}\right),\right. \\
\left.x^{\prime \prime}\left(t_{1}\right), 0\right)>0 .
\end{gathered}
$$

We may assume that $x^{\prime \prime}\left(t_{1}\right)>0$; then, $x^{(4)}\left(t_{1}\right)>0$. Thus, by the continuity of $x^{(4)}(t)$ on $[0,1]$, there exists $\delta>0$ such that $x^{(4)}(t)>0$ for $t \in\left(t_{1}-\delta, t_{1}\right] \subset[0,1]$. Since $x^{\prime \prime \prime}\left(t_{1}\right)=0$, it follows that $x^{\prime \prime \prime}(t)<0$ for $t \in\left(t_{1}-\delta, t_{1}\right]$; namely, $x^{\prime \prime}(t)$ is decreasing on $\left(t_{1}-\delta, t_{1}\right]$, which contradicts the fact that $x^{\prime \prime}(t)$ attains its positive maximum value at $t=t_{1}$. In summary, inequality (23) is true, which implies from $x(0)=x^{\prime}(1)=0$ that

$$
\left|x^{(i)}(t)\right| \leq M+r, \quad i=0,1, t \in[0,1] .
$$

This completes the proof of the lemma.

Remark 5. In Lemma 4, if condition (i) is replaced by

$\left(i^{\prime}\right)$, there exists a constant $M>0$ such that whenever $\left|x_{2}\right|>M$ and all $\left(t, x_{0}, x_{1}\right) \in[0,1] \times \mathbb{R}^{2}$,

$$
x_{2} f\left(t, x_{0}, x_{1}, x_{2}, 0\right)>0 \text {; }
$$

then, the conclusion of Lemma 4 remains true.

The following fixed point result due to D. O'Regan plays a crucial role.

Lemma 6 (see [31]). Let $U$ be an open set in a closed, convex set $C$ of a Banach space $E$. Assume that $0 \in U, P(\bar{U})$ is bounded, and $P: \bar{U} \rightarrow C$ is given by $P=P_{1}+P_{2}$, where $P_{1}: \bar{U} \rightarrow E$ is continuous and completely continuous and $P_{2}: \bar{U} \rightarrow E$ is a nonlinear contraction. Then, either

$\left(\mathrm{A}_{1}\right) P$ has a fixed point in $\bar{U}$, or

$\left(\mathrm{A}_{2}\right)$ there is a point $u \in \partial U$ and $\lambda \in(0,1)$ with $u=\lambda P(u)$.

\section{Main Results}

Firstly in this section, we state and prove our existence results of solutions for BVP (1), (2).

Theorem 7. Suppose that

(i) for each fixed $\left(t, x_{2}, x_{3}\right) \in[0,1] \times \mathbb{R}^{2}, f\left(t, x_{0}, x_{1}, x_{2}\right.$, $x_{3}$ ) is nondecreasing in $x_{0}$ and $x_{1}$;

(ii) there exists a constant $M>0$ such that for $|x|>M$, $t \in[0,1]$,

$$
x f(t,-x,-x, x, 0)>0
$$


(iii) there exist $\beta \in(0,1)$ and nondecreasing continuous function $\sigma:[0,+\infty) \rightarrow[0,+\infty)$ such that $\sigma(u) \leq \beta u$ for $u>0$, and

$$
\begin{aligned}
& \left|h\left(t, x_{0}, x_{1}, x_{2}\right)-h\left(t, y_{0}, y_{1}, y_{2}\right)\right| \\
& \quad \leq \sigma\left(\max \left\{\left|x_{i}-y_{i}\right|, i=0,1,2\right\}\right)
\end{aligned}
$$

for all $\left(t, x_{0}, x_{1}, x_{2}\right),\left(t, y_{0}, y_{1}, y_{2}\right) \in[0,1] \times \mathbb{R}^{3}$;

(iv) $f\left(t, x_{0}, x_{1}, x_{2}, x_{3}\right)$ satisfies the Nagumo condition; that is, there exists a positive-valued continuous function $\Phi(s)$ on $[0,+\infty)$ with $\int_{0}^{+\infty}(s \mathrm{~d} s / \Phi(s))=+\infty$ such that

$$
\left|f\left(t, x_{0}, x_{1}, x_{2}, x_{3}\right)\right| \leq \Phi\left(\left|x_{3}\right|\right)
$$

for all $\left(t, x_{0}, x_{1}, x_{2}, x_{3}\right) \in[0,1] \times[-M-r, M+r]^{3} \times \mathbb{R}$, where

$$
r=(1-\beta)^{-1}\|h(\cdot, 0,0,0)\|_{0}
$$

Then, BVP (1), (2) has at least one solution.

Proof. Let $x$ be a possible solution of BVP (5), (6). We now show that

$$
\left|x^{\prime \prime \prime}(t)\right| \leq N, \quad t \in[0,1]
$$

where $N:=\max \left\{N_{0}, 2(M+r)\right\}$ and $\int_{M+r}^{N_{0}}(s \mathrm{~d} s / \Phi(s))=2(M+$ $r)+1$.

Suppose that (34) is not true. Then, there exists $t_{1} \in[0,1)$ such that $\left|x^{\prime \prime \prime}\left(t_{1}\right)\right|>N$. Since $x^{\prime \prime \prime}(1)=0$, then there exists $\xi$, $\eta\left(t_{1}<\xi<\eta<1\right)$ such that

$$
\begin{aligned}
& \left|x^{\prime \prime \prime}(\xi)\right|=N, \quad\left|x^{\prime \prime \prime}(\eta)\right|=M+r, \\
& M+r<\left|x^{\prime \prime \prime}(t)\right|<N, \quad \forall t \in(\xi, \eta) .
\end{aligned}
$$

Therefore, $x^{\prime \prime \prime}(t)$ is positive or negative on $(\xi, \eta)$ by the continuity of the $x^{\prime \prime \prime}(t)$. Hence, from assumption (iv), the definition of $N$, and Lemma 4, we can get the following contradiction:

$$
\begin{aligned}
2(M+r)+1 & =\int_{M+r}^{N_{0}} \frac{s \mathrm{~d} s}{\Phi(s)} \leq \int_{M+r}^{N} \frac{s \mathrm{~d} s}{\Phi(s)} \\
& \leq\left|\int_{\eta}^{\xi} \frac{x^{\prime \prime \prime}(t) x^{(4)}(t) \mathrm{d} t}{\Phi\left(\left|x^{\prime \prime \prime}(t)\right|\right)}\right| \\
& \leq \int_{\xi}^{\eta}\left|x^{\prime \prime \prime}(t)\right| \mathrm{d} t=\left|x^{\prime \prime}(\eta)-x^{\prime \prime}(\xi)\right| \\
& \leq 2(M+r) .
\end{aligned}
$$

Therefore, inequality (34) holds.

Let $\Omega:=\left\{x \in C^{3}[0,1]:\|x\|_{C^{3}}<N+1=: R\right\}$. It follows easily from the properties of the Green function and the continuity of $f$ that the operator $P_{1}: \bar{\Omega} \rightarrow C^{3}[0,1]$ is completely continuous.
We now show that $P_{2}: \bar{\Omega} \rightarrow C^{3}[0,1]$ is a nonlinear contraction. In fact, from assumption (iii), we have

$$
\begin{array}{r}
|(H x)(t)-(H y)(t)| \leq \sigma\left(\|x-y\|_{C^{3}}\right), \\
t \in[0,1], \forall x, y \in C^{3}[0,1] .
\end{array}
$$

Consequently, from Lemma 3, we have

$$
\begin{aligned}
&|\varphi(t, x(s))-\varphi(t, y(s))|=|g(t)(H x)(s)-g(t)(H y)(s)| \\
& \leq\|g\|_{0} \sigma\left(\|x-y\|_{C^{3}}\right) \\
& \leq \frac{1}{2} \beta\|x-y\|_{C^{3}}, \quad t, s \in[0,1], \\
& \forall x, y \in C^{3}[0,1] .
\end{aligned}
$$

Similarly, for all $x, y \in C^{3}[0,1]$,

$$
\begin{aligned}
&\left|\frac{\partial \varphi(t, x(s))}{\partial t}-\frac{\partial \varphi(t, y(s))}{\partial t}\right| \leq\left\|g^{\prime}\right\|_{0} \sigma\left(\|x-y\|_{C^{3}}\right) \\
& \leq \beta\|x-y\|_{C^{3}}, \quad t, s \in[0,1], \\
&\left|\frac{\partial^{2} \varphi(t, x(s))}{\partial t^{2}}-\frac{\partial^{2} \varphi(t, y(s))}{\partial t^{2}}\right| \leq\left\|g^{\prime \prime}\right\|_{0} \sigma\left(\|x-y\|_{C^{3}}\right) \\
& \leq \beta\|x-y\|_{C^{3}}, \quad t, s \in[0,1], \\
&\left|\frac{\partial^{3} \varphi(t, x(s))}{\partial t^{3}}-\frac{\partial^{3} \varphi(t, y(s))}{\partial t^{3}}\right|=0, \quad t, s \in[0,1] .
\end{aligned}
$$

Hence,

$$
\begin{aligned}
\| P_{2} x & -P_{2} y \|_{C^{3}} \\
& =\max \left\{\left\|\left(P_{2} x\right)^{(i)}-\left(P_{2} y\right)^{(i)}\right\|_{0}, i=0,1,2,3\right\} \\
& \leq \beta\|x-y\|_{C^{3}}, \quad \forall x, y \in C^{3}[0,1] .
\end{aligned}
$$

Since all possible solutions of BVP (5), (6) satisfy $\|x\|_{C^{3}} \leq$ $N<R$, it follows that there is no $x \in \partial \Omega$ and $\lambda \in(0,1)$ such that $x=\lambda L^{-1} T x$. We conclude that $\left(\mathrm{A}_{2}\right)$ of Lemma 6 does not hold. Consequently, $L^{-1} T=P_{1}+P_{2}$ has a fixed point, which is a solution of BVP (1), (2). This completes the proof of the theorem.

Remark 8. In Theorem 7, if condition (i) is replaced by

$\left(i^{\prime}\right)$ there exists a constant $M>0$ such that whenever $\left|x_{2}\right|>M$ and all $\left(t, x_{0}, x_{1}\right) \in[0,1] \times \mathbb{R}^{2}$,

$$
x_{2} f\left(t, x_{0}, x_{1}, x_{2}, 0\right)>0 \text {, }
$$

then the conclusion of Theorem 7 remains true.

Remark 9. In Theorem 7 , if $f \geq 0 \geq h$ and $f(t, 0,0,0,0) \not \equiv 0$, then all the solutions of BVP (1), (2) are monotone and positive. This is clear because by Lemma 3 we have $x=P_{1} x+P_{2} x \geq$ $0, x^{\prime}=\left(P_{1} x\right)^{\prime}+\left(P_{2} x\right)^{\prime} \geq 0$ and $x^{\prime \prime}=\left(P_{1} x\right)^{\prime \prime}+\left(P_{2} x\right)^{\prime \prime} \leq 0$.

Next, we consider the existence of solutions and positive solutions for BVP (3), (4). 
Theorem 10. Suppose that

(i) there exists $\Psi:[0,+\infty) \rightarrow[0,+\infty)$ being continuous and nondecreasing such that

$$
|f(t, x)| \leq \Psi(|x|), \quad \forall(t, x) \in[0,1] \times \mathbb{R} ;
$$

(ii) there exists $\beta \in(0,2)$ such that

$$
\begin{aligned}
& |h(t, x)-h(t, y)| \\
& \quad \leq \beta|x-y|, \quad \forall(t, x, y) \in[0,1] \times \mathbb{R}^{2} ;
\end{aligned}
$$

(iii) there exists $\gamma>0$ such that

$$
\frac{\Psi(\gamma)+(3 / 2) h_{0}}{\gamma}<3\left(1-\frac{1}{2} \beta\right),
$$

where $h_{0}=\max _{0 \leq t \leq 1}|h(t, 0)|$.

Then, BVP (3), (4) has at least one solution.

Proof. It is easy to see that $x \in C^{4}[0,1]$ is a solution of BVP (3), (4) if and only if $x \in C[0,1]$ is a solution of the integral equation (14) with $\lambda=1$. Moreover, $P_{1}$ is completely continuous, and $P_{2}$ is a nonlinear contraction.

It follows from (15), (i), and Lemma 3 that $\forall x \in C[0,1]$,

$$
\begin{aligned}
\left|\left(P_{1} x\right)(t)\right| & \leq \int_{0}^{1} G(t, s) \Psi(|x(s)|) \mathrm{d} s \\
& \leq \frac{1}{3} \Psi\left(\|x\|_{0}\right), \quad t \in[0,1] .
\end{aligned}
$$

Also, (16), (ii), and Lemma 3 yield

$$
\begin{aligned}
\left|\left(P_{2} x\right)(t)\right| & \leq \int_{0}^{1}|g(t)(H x)(s)| \mathrm{d} s \\
& \leq\|g\|_{0} \int_{0}^{1}(|h(s, x(s))-h(s, 0)|+|h(s, 0)|) \mathrm{d} s \\
& \leq \frac{1}{2}\left(\beta\|x\|_{0}+h_{0}\right), \quad \forall x \in C[0,1], t \in[0,1] .
\end{aligned}
$$

From (14), (45), and (46), we have that all possible solutions of $x=\lambda\left(P_{1}+P_{2}\right) x$ satisfy

$$
|x(t)| \leq \frac{1}{3} \Psi\left(\|x\|_{0}\right)+\frac{1}{2}\left(\beta\|x\|_{0}+h_{0}\right), \quad t \in[0,1] .
$$

Let $\Omega:=\left\{x \in C[0,1]:\|x\|_{0}<\gamma\right\}$. Then, $\Omega$ is open in $C[0,1], 0 \in \Omega$, and $\left(P_{1}+P_{2}\right)(\bar{\Omega})$ is bounded. Suppose that $x \in \partial \Omega$ and $\lambda \in(0,1)$ satisfy $x=\lambda\left(P_{1}+P_{2}\right) x$. Then, $\|x\|_{0}=\gamma$, and (i) and (47) lead to

$$
\gamma \leq \frac{1}{3} \Psi(\gamma)+\frac{1}{2}\left(\beta \gamma+h_{0}\right)
$$

that is,

$$
\frac{\Psi(\gamma)+(3 / 2) h_{0}}{\gamma} \geq 3\left(1-\frac{1}{2} \beta\right),
$$

which contradicts (iii). Hence, $\left(\mathrm{A}_{2}\right)$ of Lemma 6 does not hold, and consequently $L^{-1} T=P_{1}+P_{2}$ has a fixed point which is a solution of BVP (3), (4). This completes the proof of the theorem.

Remark 11. In Theorem 10, if $f \geq 0 \geq h$ and $f(t, 0) \neq \equiv 0$, then all the solutions of BVP (3), (4) are monotone and positive.

Now, we consider BVP (3), (4) with linear boundary conditions as

$$
h(t, x(t))=l(t) x(t), \quad t \in[0,1],
$$

where $l(\cdot) \in C[0,1]$. Define

$$
K(t, s)=g(t) l(s), \quad(t, s) \in[0,1] \times[0,1] .
$$

Then,

$$
\left(P_{2} x\right)(t)=\int_{0}^{1} K(t, s) x(s) \mathrm{d} s
$$

Theorem 12. Suppose that

(i) $K_{0}:=(1 / 2) \int_{0}^{1}|l(s)| \mathrm{d} s<1$;

(ii) there exists $\Psi:[0,+\infty) \rightarrow[0,+\infty)$ being continuous and nondecreasing such that

$$
\begin{gathered}
|f(t, x)| \leq \Psi(|x|), \quad \forall(t, x) \in[0,1] \times \mathbb{R}, \\
\limsup _{\rho \rightarrow+\infty} \frac{\Psi(\rho)}{\rho}<3\left(1-K_{0}\right) .
\end{gathered}
$$

Then, the nonlinear fourth-order differential equation (3) with boundary conditions

$$
\begin{gathered}
x(0)=x^{\prime}(1)=0, \\
x^{\prime \prime}(0)=\int_{0}^{1} l(s) x(s) \mathrm{d} s, \\
x^{\prime \prime \prime}(1)=0
\end{gathered}
$$

has at least one solution.

Proof. Notice that the existence of solutions of BVP (3), (54) is equivalent to the existence of fixed points of operator equation

$$
x=P_{1} x+P_{2} x .
$$

As a linear operator on $C[0,1]$, from (52) and (i), we get $\left\|P_{2}\right\|=K_{0}<1$, which implies that $I-P_{2}$ is invertible and its inverse is given by

$$
\left(I-P_{2}\right)^{-1}=\sum_{n=0}^{\infty} P_{2}^{n} \quad \text { with }\left\|\left(I-P_{2}\right)^{-1}\right\| \leq \frac{1}{1-K_{0}} .
$$

Hence, we see from (55) that $x$ is a solution of BVP (3), (54) if and only if $x$ is a fixed point of the completely continuous operator $S=\left(I-P_{2}\right)^{-1} P_{1}$. 
Let us show that there exists $\rho^{*}>0$ such that any solution $x$ of operator equation $x=\lambda S x(\lambda \in(0,1))$ satisfies $\|x\|_{0}<\rho^{*}$. In fact, any solution $x$ of $x=\lambda S x(\lambda \in(0,1))$ satisfies

$$
x(t)=\lambda\left(I-P_{2}\right)^{-1} \int_{0}^{1} G(t, s) f(s, x(s)) \mathrm{d} s,
$$

and, hence, by (ii) and Lemma 3, we have

$$
\|x\|_{0} \leq \frac{1}{3\left(1-K_{0}\right)} \Psi\left(\|x\|_{0}\right) .
$$

The condition lim $\sup _{\rho \rightarrow+\infty}(\Psi(\rho) / \rho)<3\left(1-K_{0}\right)$ implies that there exists $\rho^{*}>0$ such that $(\Psi(\rho) / \rho)<3\left(1-K_{0}\right)$ for all $\rho \geq \rho^{*}$; that is,

$$
\rho>\frac{1}{3\left(1-K_{0}\right)} \Psi(\rho), \quad \forall \rho \geq \rho^{*} .
$$

Comparing (58) and (59), we see that $\|x\|_{0}<\rho^{*}$.

Now, let $\Omega=\left\{x \in C[0,1]:\|x\|_{0}<\rho^{*}\right\}$. By the LeraySchauder continuation theorem $S$ has a fixed point in $\Omega$, which is a solution of BVP (3), (54). This completes the proof of the theorem.

Remark 13. In Theorem 12, if $f \geq 0 \geq l$ and $f(t, 0) \quad \equiv \equiv 0$, then all the solutions of BVP (3), (54) are monotone and positive since all the solutions of BVP (3), (54) satisfy

$$
x(t)=\left(\sum_{n=0}^{\infty} P_{2}^{n}\right) \int_{0}^{1} G(t, s) f(s, x(s)) \mathrm{d} s \geq 0 \quad \text { on }[0,1],
$$

and thus

$$
x^{\prime}=\left(P_{1} x\right)^{\prime}+\left(P_{2} x\right)^{\prime} \geq 0, \quad x^{\prime \prime}=\left(P_{1} x\right)^{\prime \prime}+\left(P_{2} x\right)^{\prime \prime} \leq 0 .
$$

Finally, we give some examples to illustrate our results.

Example 14. Consider the fourth-order boundary value problem

$$
\begin{gathered}
x^{(4)}(t)=t^{2}(x(t))^{3}+\frac{1}{2}\left(x^{\prime}(t)\right)^{5} \\
+x^{\prime \prime}(t) e^{\left(x^{\prime \prime}(t)\right)^{2}}+\left(x^{\prime \prime \prime}(t)\right)^{2}, \quad t \in[0,1], \\
x(0)=x^{\prime}(1)=0, \\
x^{\prime \prime}(0)=\frac{1}{2} \int_{0}^{1} \sqrt{1+\left(x^{\prime \prime}(s)\right)^{2}} \mathrm{~d} s, \\
x^{\prime \prime \prime}(1)=0 .
\end{gathered}
$$

Let

$$
\begin{gathered}
f\left(t, x_{0}, x_{1}, x_{2}, x_{3}\right)=t^{2} x_{0}^{3}+\frac{1}{2} x_{1}^{5}+x_{2} e^{x_{2}^{2}}+x_{3}^{2} \\
\text { on }[0,1] \times \mathbb{R}^{4}, \\
h\left(t, x_{0}, x_{1}, x_{2}\right)=\frac{1}{2} \sqrt{1+x_{2}^{2}} \quad \text { on }[0,1] \times \mathbb{R}^{3}, \\
\sigma(u)=\frac{1}{2} u \quad \text { on }[0,+\infty) .
\end{gathered}
$$

It is easy to check that all the assumptions in Theorem 7 are satisfied. Hence, BVP (62), (63) has at least one solution.

Example 15. Consider the fourth-order boundary value problem

$$
\begin{gathered}
x^{(4)}(t)=q(t)(x(t))^{2}+1, \quad t \in[0,1], \\
x(0)=x^{\prime}(1)=0, \\
x^{\prime \prime}(0)=\frac{3}{2} \int_{0}^{1} \ln \frac{1}{1+(x(s))^{2}} \mathrm{~d} s, \\
x^{\prime \prime \prime}(1)=0,
\end{gathered}
$$

where $q(t) \in C([0,1],[0,(1 / 8)])$.

Let

$$
\begin{gathered}
f(t, x)=q(t) x^{2}+1 \quad \text { on }[0,1] \times \mathbb{R}, \\
h(t, x)=\frac{3}{2} \ln \frac{1}{1+x^{2}} \quad \text { on }[0,1] \times \mathbb{R}, \\
\Psi(x)=\frac{1}{8} x^{2}+1 \quad \text { on }[0,+\infty), \\
\beta=\frac{3}{2}, \quad \gamma=3 .
\end{gathered}
$$

It is easy to check that all the assumptions in Theorem 10 and Remark 11 are satisfied. Hence, BVP (65), (66) has at least one monotone positive solution.

Example 16. Consider the fourth-order boundary value problem

$$
\begin{gathered}
x^{(4)}(t)=q(t) \sqrt[3]{(x(t))^{2}}+1, \quad t \in[0,1], \\
x(0)=x^{\prime}(1)=0, \\
x^{\prime \prime}(0)=\int_{0}^{1}\left(s^{2}-4 s\right) x(s) \mathrm{d} s \\
x^{\prime \prime \prime}(1)=0
\end{gathered}
$$

where $q(t) \in C([0,1],[0,+\infty))$.

Let

$$
\begin{gathered}
f(t, x)=q(t) \sqrt[3]{x^{2}}+1 \quad \text { on }[0,1] \times \mathbb{R}, \\
\Psi(x)=\left(\max _{t \in[0,1]} q(t)\right) \sqrt[3]{x^{2}}+1 \quad \text { on }[0,+\infty), \\
l(t)=t^{2}-4 t \quad \text { on }[0,1] .
\end{gathered}
$$

It is easy to check that all the assumptions in Theorem 12 and Remark 13 are satisfied. Hence, BVP (68), (69) has at least one monotone positive solution.

\section{Acknowledgment}

This work was supported by NSFC (11126339). 


\section{References}

[1] R. P. Agarwal and Y. M. Chow, "Iterative methods for a fourth order boundary value problem," Journal of Computational and Applied Mathematics, vol. 10, no. 2, pp. 203-217, 1984.

[2] Z. Bai, "The upper and lower solution method for some fourthorder boundary value problems," Nonlinear Analysis. Theory, Methods \& Applications, vol. 67, no. 6, pp. 1704-1709, 2007.

[3] A. Cabada and F. M. Minhós, "Fully nonlinear fourth-order equations with functional boundary conditions," Journal of Mathematical Analysis and Applications, vol. 340, no. 1, pp. 239251, 2008.

[4] M. A. Del Pino and R. F. Manásevich, "Existence for a fourthorder boundary value problem under a two-parameter nonresonance condition," Proceedings of the American Mathematical Society, vol. 112, no. 1, pp. 81-86, 1991.

[5] J. Du and M. Cui, "Constructive proof of existence for a class of fourth-order nonlinear BVPs," Computers \& Mathematics with Applications, vol. 59, no. 2, pp. 903-911, 2010.

[6] J. Ehme, P. W. Eloe, and J. Henderson, "Upper and lower solution methods for fully nonlinear boundary value problems," Journal of Differential Equations, vol. 180, no. 1, pp. 51-64, 2002.

[7] D. Franco, D. O’Regan, and J. Perán, "Fourth-order problems with nonlinear boundary conditions," Journal of Computational and Applied Mathematics, vol. 174, no. 2, pp. 315-327, 2005.

[8] H. Feng, D. Ji, and W. Ge, "Existence and uniqueness of solutions for a fourth-order boundary value problem," Nonlinear Analysis. Theory, Methods \& Applications, vol. 70, no. 10, pp. 3561-3566, 2009.

[9] J. R. Graef and L. Kong, "A necessary and sufficient condition for existence of positive solutions of nonlinear boundary value problems," Nonlinear Analysis. Theory, Methods \& Applications, vol. 66, no. 11, pp. 2389-2412, 2007.

[10] M. R. Grossinho and St. A. Tersian, "The dual variational principle and equilibria for a beam resting on a discontinuous nonlinear elastic foundation," Nonlinear Analysis. Theory, Methods \& Applications, vol. 41, no. 3-4, pp. 417-431, 2000.

[11] C. P. Gupta, "Existence and uniqueness theorems for the bending of an elastic beam equation," Applicable Analysis, vol. 26, no. 4, pp. 289-304, 1988.

[12] T. Jankowski, "Positive solutions for fourth-order differential equations with deviating arguments and integral boundary conditions," Nonlinear Analysis. Theory, Methods \& Applications, vol. 73, no. 5, pp. 1289-1299, 2010.

[13] D. Jiang, W. Gao, and A. Wan, "A monotone method for constructing extremal solutions to fourth-order periodic boundary value problems," Applied Mathematics and Computation, vol. 132, no. 2-3, pp. 411-421, 2002.

[14] P. Kang, Z. Wei, and J. Xu, "Positive solutions to fourthorder singular boundary value problems with integral boundary conditions in abstract spaces," Applied Mathematics and Computation, vol. 206, no. 1, pp. 245-256, 2008.

[15] Y. Li, "Positive solutions of fourth-order boundary value problems with two parameters," Journal of Mathematical Analysis and Applications, vol. 281, no. 2, pp. 477-484, 2003.

[16] X.-L. Liu and W.-T. Li, "Existence and multiplicity of solutions for fourth-order boundary value problems with three parameters," Mathematical and Computer Modelling, vol. 46, no. 3-4, pp. 525-534, 2007.

[17] Y. Liu, "Multiple positive solutions of nonlinear singular boundary value problem for fourth-order equations," Applied Mathematics Letters, vol. 17, no. 7, pp. 747-757, 2004.
[18] S. Lu and S. Jin, "Existence of periodic solutions for a fourthorder P-Laplacian equation with a deviating argument," Journal of Computational and Applied Mathematics, vol. 230, no. 2, pp. 513-520, 2009.

[19] T. F. Ma, "Positive solutions for a beam equation on a nonlinear elastic foundation," Mathematical and Computer Modelling, vol. 39, no. 11-12, pp. 1195-1201, 2004.

[20] R. Ma and J. Xu, "Bifurcation from interval and positive solutions of a nonlinear fourth-order boundary value problem," Nonlinear Analysis. Theory, Methods \& Applications, vol. 72, no. 1, pp. 113-122, 2010.

[21] R. Ma and T. Chen, "Existence of positive solutions of fourthorder problems with integral boundary conditions," Boundary Value Problems, vol. 2011, Article ID 297578, 17 pages, 2011.

[22] F. Minhós, T. Gyulov, and A. I. Santos, "Lower and upper solutions for a fully nonlinear beam equation," Nonlinear Analysis. Theory, Methods \& Applications, vol. 71, no. 1-2, pp. 281-292, 2009.

[23] P. K. Palamides, "Uniqueness of monotone positive solutions for singular boundary value problems," Communications on Applied Nonlinear Analysis, vol. 9, no. 3, pp. 79-89, 2002.

[24] P. Pietramala, "A note on a beam equation with nonlinear boundary conditions," Boundary Value Problems, vol. 2011, Article ID 376782, 14 pages, 2011.

[25] M. Pei and S. K. Chang, "Monotone iterative technique and symmetric positive solutions for a fourth-order boundary value problem," Mathematical and Computer Modelling, vol. 51, no. 910, pp. 1260-1267, 2010.

[26] J. R. L. Webb, G. Infante, and D. Franco, "Positive solutions of nonlinear fourth-order boundary-value problems with local and non-local boundary conditions," Proceedings of the Royal Society of Edinburgh A, vol. 138, no. 2, pp. 427-446, 2008.

[27] Z. Wei, "A class of fourth order singular boundary value problems," Applied Mathematics and Computation, vol. 153, no. 3, pp. 865-884, 2004.

[28] Q. Yao, "Positive solutions for eigenvalue problems of fourthorder elastic beam equations," Applied Mathematics Letters, vol. 17, no. 2, pp. 237-243, 2004.

[29] X. Zhang and W. Ge, "Positive solutions for a class of boundaryvalue problems with integral boundary conditions," Computers \& Mathematics with Applications, vol. 58, no. 2, pp. 203-215, 2009.

[30] A. Boucherif, S. M. Bouguima, N. Al-Malki, and Z. Benbouziane, "Third order differential equations with integral boundary conditions," Nonlinear Analysis. Theory, Methods \& Applications, vol. 71, no. 12, pp. el736-e1743, 2009.

[31] D. O'Regan, "Fixed-point theory for the sum of two operators," Applied Mathematics Letters, vol. 9, no. 1, pp. 1-8, 1996. 


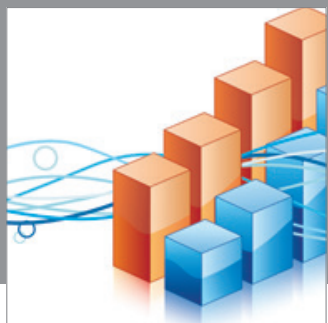

Advances in

Operations Research

mansans

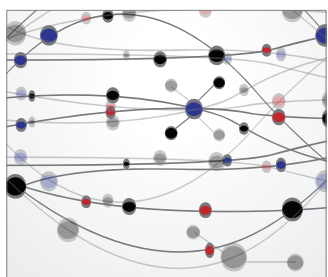

The Scientific World Journal
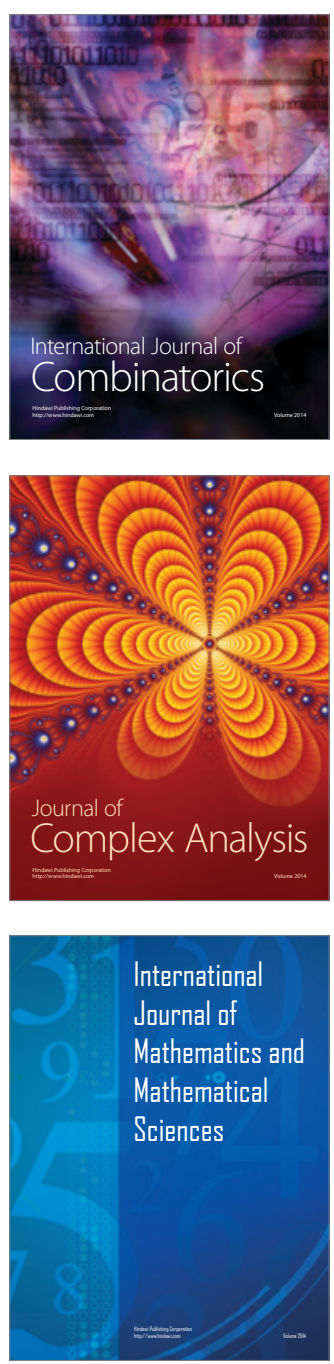
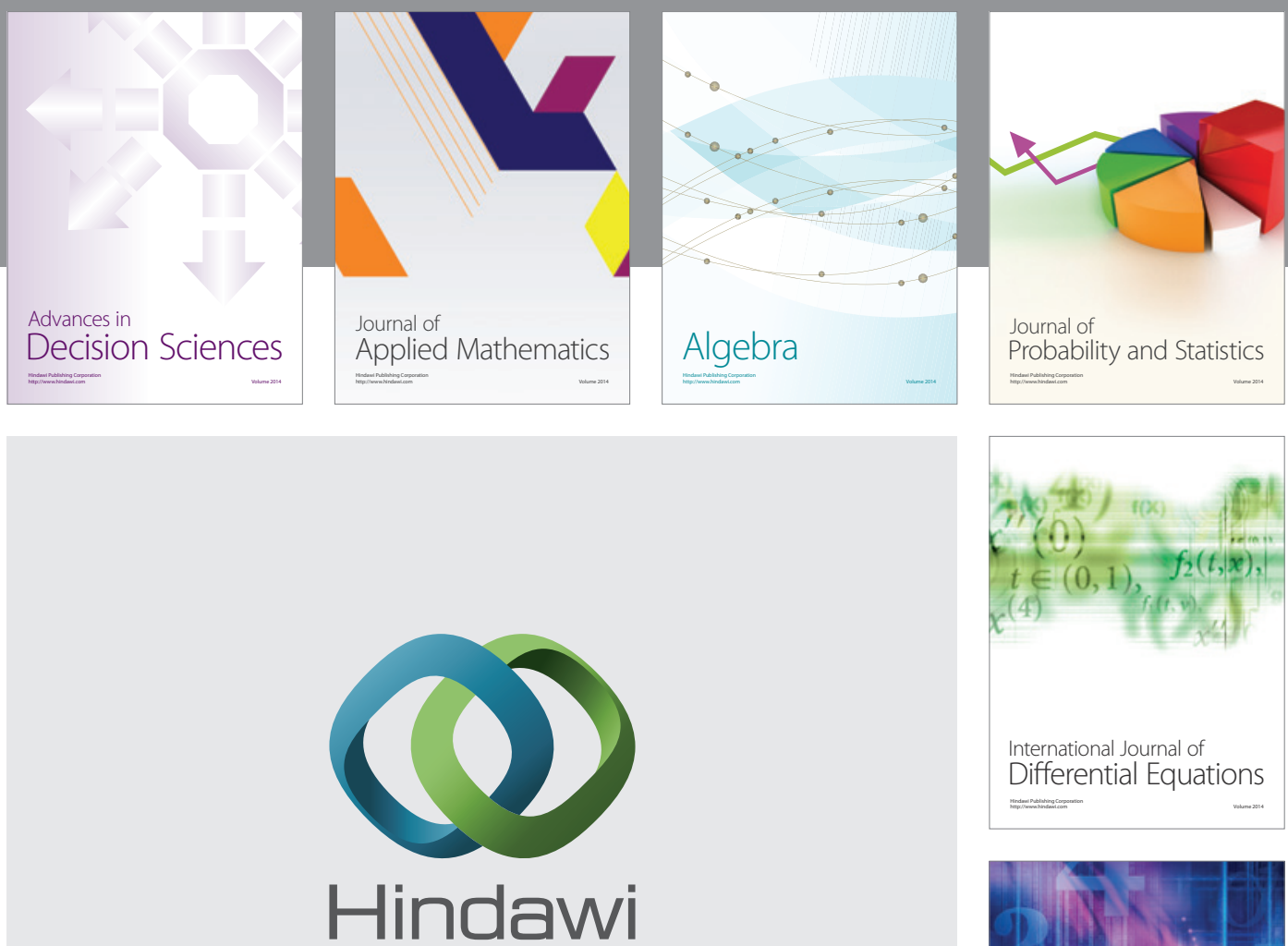

Submit your manuscripts at http://www.hindawi.com
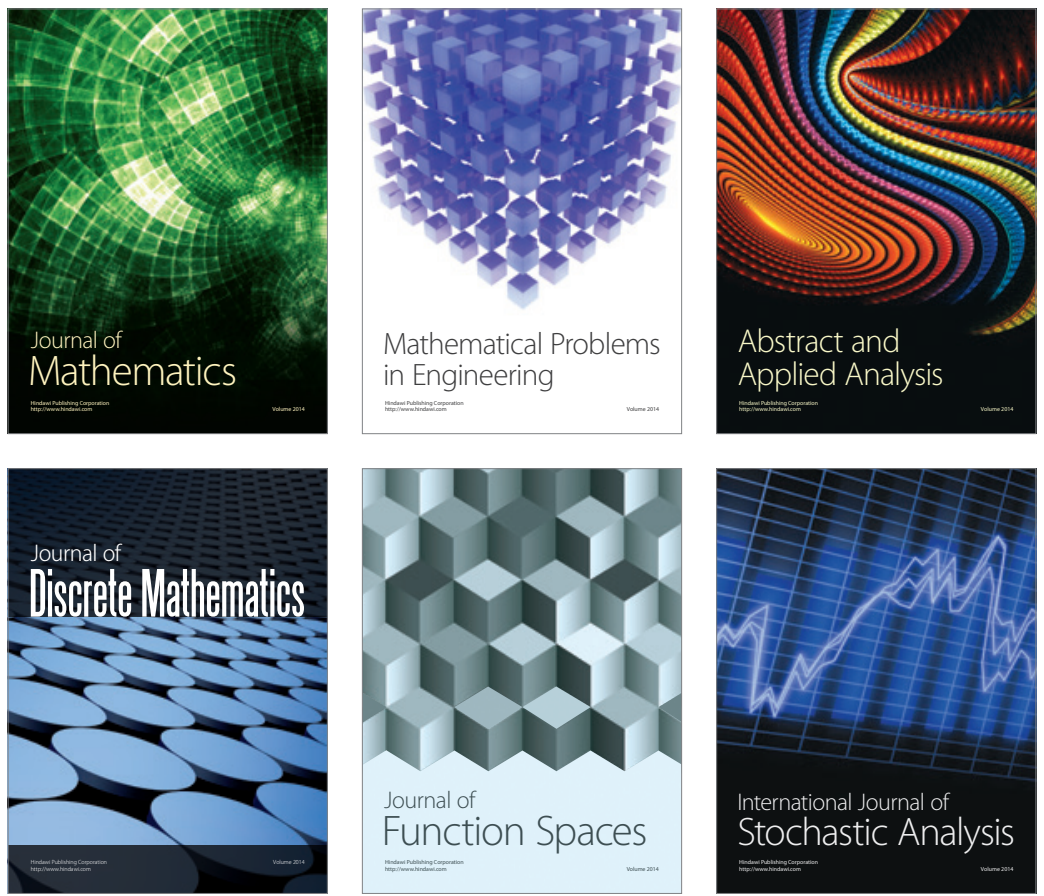

Journal of

Function Spaces

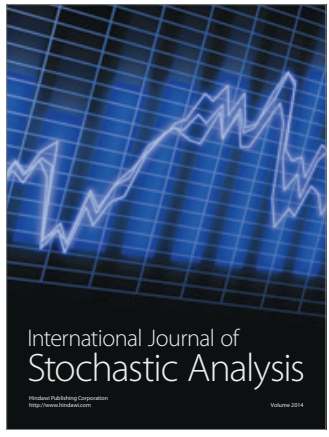

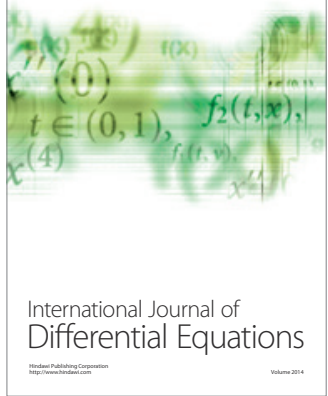
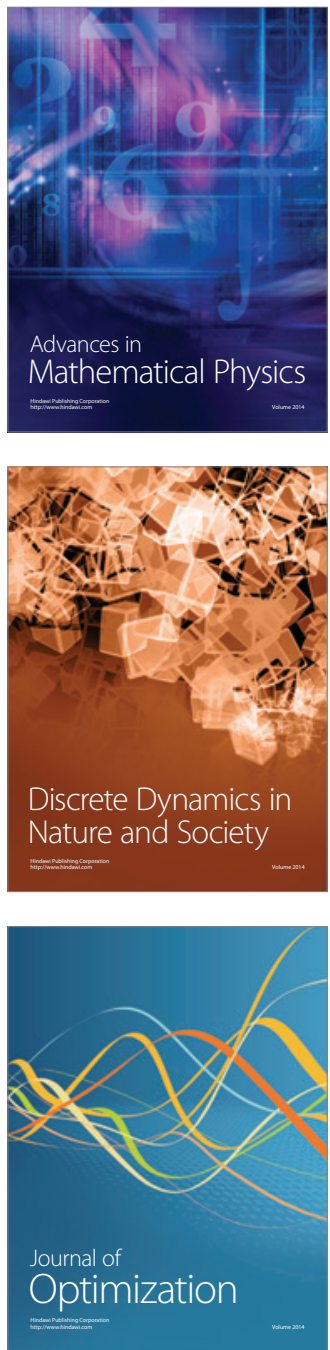